\title{
Temperature-dependent linewidth of charged excitons in semiconductor quantum dots: Strongly broadened ground state transitions due to acoustic phonon scattering
}

\author{
B. Urbaszek, ${ }^{1,2}$ E. J. McGhee, ${ }^{1}$ M. Krüger, ${ }^{1}$ R. J. Warburton, ${ }^{1}$ K. Karrai ${ }^{3}$ T. Amand, ${ }^{2}$ B. D. Gerardot, ${ }^{4}$ \\ P. M. Petroff, ${ }^{4}$ and J. M. Garcia ${ }^{5}$ \\ ${ }^{1}$ School of Engineering and Physical Sciences, Heriot Watt University, Edinburgh EH14 4AS, United Kingdom \\ ${ }^{2}$ Laboratoire de Physique de la Matière Condensée, LNMO-INSA-CNRS, 135 Avenue de Rangueil, 31077 Toulouse Cedex 4, France \\ ${ }^{3}$ Center for NanoScience and Sektion Physik, Ludwig-Maximilians-Universität,Geschwister-Scholl-Platz, 1, 80539 München, Germany \\ ${ }^{4}$ Materials Department and QUEST, University of California, Santa Barbara, California 93106, USA \\ ${ }^{5}$ Instituto de Microelectrónica de Madrid, CNM-CSIC Isaac Newton 8, PTM, 28760 Tres Cantos, Madrid, Spain
}

(Received 22 July 2003; published 8 January 2004)

\begin{abstract}
We report temperature-dependent photoluminescence on neutral and charged excitons in individual InAs quantum dots. We find narrow emission lines for temperatures up to $30 \mathrm{~K}$ for exciton transitions where only the electron ground state is occupied. In contrast, for doubly charged excitons where the excited electron state is occupied, we observe a drastic increase of the ground state transition linewidth even at $30 \mathrm{~K}$. We interpret this as evidence that the excited electron state is degenerate with the low energy tail of continuum states.
\end{abstract}

DOI: 10.1103/PhysRevB.69.035304

PACS number(s): 73.21.La, 78.55.Cr, 78.67.Hc

Many optical properties of self-assembled semiconductor quantum dots have been successfully described with the artificial atom model. ${ }^{1}$ Spatial confinement of electrons and holes in three directions gives rise to a shell structure that is observed as a quantum dot is filled with either excitons ${ }^{2}$ or electrons. ${ }^{3}$ Due to the discrete nature of the transitions, interactions of the quantum dot with its solid state environment are strongly suppressed and long optical dephasing times ${ }^{4}$ and spin relaxation quenching ${ }^{5}$ have been observed recently at low temperature. The rapid progress of devices using quantum dots as single photon emitters ${ }^{6-9}$ serves as an example for possible implementations of these nanostructures in future spintronics and quantum information applications.

Temperature dependent photoluminescence (PL) studies on single InAs (Refs. 10-12) and CdTe (Ref. 13) quantum dots have shown a strongly reduced broadening of the ground state transition linewidth compared to semiconductor quantum wells or bulk, as predicted by the artificial atom model. In contrast, temperature dependent photoluminescence-excitation (PLE) studies of excited transitions $^{14,15}$ in single dots have shown an unexpected broadening of the transition lines. Taking into account only the discrete states in an artificial atom picture fails to explain the observed behavior. Instead, it is thought that a degeneracy between bound state transitions and transitions involving bound and delocalized states ${ }^{16}$ is responsible for the measured broadening. A detailed analysis of the underlying mechanisms for the broadening of the transitions in the PLE experiments is difficult, as the exact configuration of the excited electron and hole states investigated is not known. A thorough investigation of the limitations of the artificial atom picture and therefore the extent to which the quantum dot properties are influenced by the surrounding semiconductor matrix or the existence of continuum states in general will shed light on the feasibility of devices based on discrete quantum dot states.

Here we report on the change in PL linewidth as a function of temperature for excitons in single semiconductor quantum dots as they are charged with excess electrons. Our approach is to compare configurations with different occupations of $s$ - and $p$-like electron levels. We do this by adding excess electrons to the neutral exciton $X^{0}$ to create the singly charged exciton $X^{1-}$ and the doubly charged exciton $X^{2-}$, and so on. For each charge state we monitor the emission from the ground state in single dot PL experiments, so that no excited hole states are involved. For $X^{2-}$, the electron level above the $s$-like ground state, the $p$ level, is occupied. We show that for a temperature change from 4 to $30 \mathrm{~K}$, a small linear increase in the PL transition linewidth for the $X^{0}$ and the $X^{1-}$ transitions occurs. In contrast, for the $X^{2-}$ emission the linewidth from the same dot changes drastically over the same temperature range. We argue that this occurs through acoustic phonon scattering between the $p$ state and an energetically adjacent band of states.

We employ quantum dots embedded in a field-effect structure that allows us to control precisely the charge of the exciton. ${ }^{3}$ In these experiments, a hole is generated with optical excitation. Over large regions of bias voltage, the excitonic charge is constant, and there are abrupt steps in emission energy whenever an additional electron is added to the $\operatorname{dot}^{3}{ }^{3}$ The sample is grown on a GaAs substrate, on which a buffer layer, an $n^{+}$-GaAs layer (the back contact) and then a $25 \mathrm{~nm}$ thick undoped GaAs layer (the tunnel barrier) are grown. Subsequently, the InAs quantum dots are formed in the Stranski-Krastanow growth mode, with a density in the range of $10^{9} \mathrm{~cm}^{-2}$. A GaAs/AlAs superlattice (the blocking barrier), grown on top of the GaAs covering the dots, ensures that the coupling between the dots and a surface gate electrode is purely capacitive. The dots have either an ensemble PL centered at $1.3 \mathrm{eV}$ (sample A) or at $1.1 \mathrm{eV}$ (sample B) depending on the growth. We have performed PL spectroscopy of individual quantum dots as a function of gate voltage $V_{g}$ at different temperatures using a confocal microscope. The PL is excited with an $850 \mathrm{~nm}$ laser diode, and is dispersed and detected with a $0.5 \mathrm{~m}$ focal length spectrometer$\mathrm{Si}-\mathrm{CCD}$ system. The spectral resolution of our spectrometerdetector system is $\Gamma_{\text {res }}=0.05 \mathrm{meV}$. This is the linewidth at 


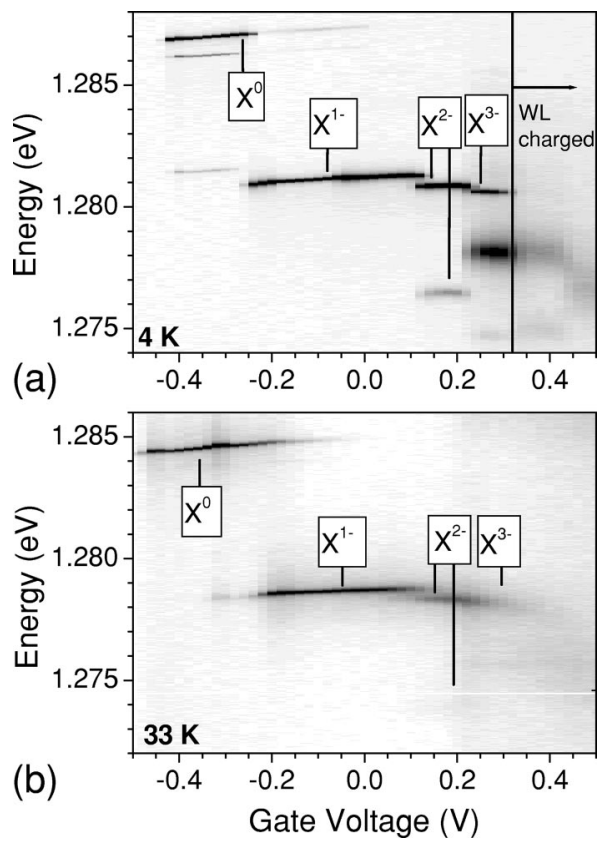

FIG. 1. (a) Gray-scale plot of the photoluminescence versus gate voltage for a single dot from sample A at $4.2 \mathrm{~K}$. White corresponds to 0 counts, black to 1000 counts on the detector. The excitons are labeled with $X$ standing for exciton, with excess charge as the suffix. Above a voltage of about $0.32 \mathrm{~V}$, the wetting layer (WL) loads with electrons. The unlabeled weaker PL lines are related to biexciton emission. (b) Measurement at $33 \mathrm{~K}$ for the same dot.

FWHM as measured with a source with a linewidth known to be orders of magnitude smaller than our spectral resolution, i.e., an ultranarrow laser source. A transition linewidth can be determined by deconvolution with a greater precision than the spectral resolution and for our experiments the minimum precision is $0.02 \mathrm{meV}$. The precision is determined by the typical signal noise and also by the pixel size of the detector. Here we are aiming to obtain values for the intrinsic transition linewidths $\Gamma$. This can be achieved by correcting the measured linewidth $\Gamma_{m}$ by the spectral resolution $\Gamma_{\text {res }}$ of our experiment as given by $\Gamma_{m}^{2}=\Gamma_{\text {res }}^{2}+\Gamma^{2}$. All linewidths discussed in the following refer to the values for the transition linewidth $\Gamma$. At low laser power, for each individual dot, we can identify $X^{0}, X^{1-}, X^{2-}$, and $X^{3-}$ by the large jump in PL energy on going from $X^{0}$ to $X^{1-}$, and the characteristic splittings of $X^{2-}$ and $X^{3-}$, as shown in Fig. 1 for a dot from sample A.

The neutral and charged exciton transitions seen at $4.2 \mathrm{~K}$ in Fig. 1(a) can still be distinguished at $33 \mathrm{~K}$ in Fig. 1(b). The gate voltage range over which the individual transitions dominate as well as the PL energy differences between the transitions remain unchanged. At $4.2 \mathrm{~K}$ the change from $X^{0}$ to $X^{1-}$ takes place over a gate voltage range of $\sim 20 \mathrm{mV}$ corresponding to an energy range of $3 \mathrm{meV}^{17}$ At $33 \mathrm{~K}, X^{0}$ and $X^{1-}$ coexist over a larger gate voltage range of $\sim 100 \mathrm{mV}$ (energy range of $14 \mathrm{meV}$ ) and this is caused by thermal occupation of the exciton with a higher energy state.

Taking into account the spectral resolution of our experiment we obtain for the neutral exciton $X^{0}$ a linewidth $\Gamma$ of $10 \pm 20 \mu \mathrm{eV}$ at $4.2 \mathrm{~K}$, in good agreement with the recent
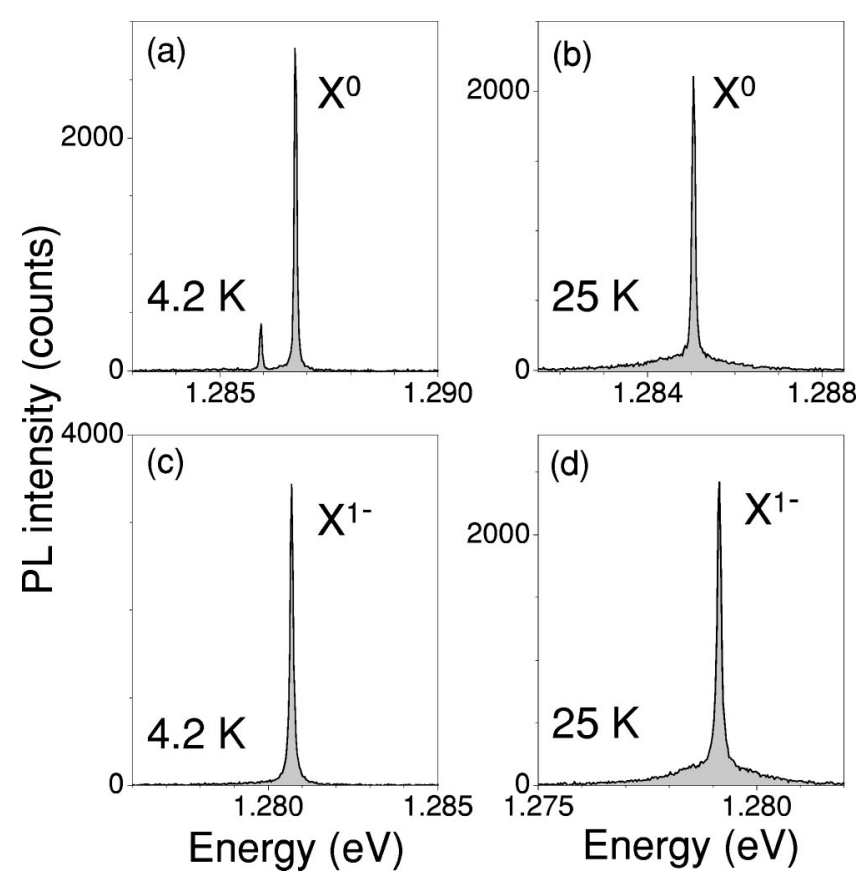

FIG. 2. PL of individual exciton transitions from sample A. (a) The neutral exciton $X^{0}$ at $4.2 \mathrm{~K}$, the smaller peak on the low energy side is attributed to the biexciton. (b) $X^{0}$ at $25 \mathrm{~K}$, (c) the $X^{1-}$ transition at $4.2 \mathrm{~K}$, and (d) $X^{1-}$ at $25 \mathrm{~K}$.

results of a transmission experiment with a narrow linewidth laser. ${ }^{18}$ This value increases only by a few $\mu \mathrm{eV}$ as the temperature is raised to $30 \mathrm{~K}$. The main effect of the temperature increase on the $X^{0}$ emission line is the strong deviation from a Lorentzian line shape at higher temperature, as shown in Fig. 2. This effect has been observed by Besombes et al. ${ }^{13}$ for CdTe dots and by Borri et al. ${ }^{4}$ for In As dots and has been attributed to lattice relaxation due to exciton-acoustic phonon coupling. For the temperature range investigated, optical phonon broadening is negligible and the measured transition linewidths follow a linear dependence typical of acoustic phonon scattering

$$
\Gamma(T)=\Gamma_{0}+\alpha T,
$$

where $\Gamma_{0}$ is the transition linewidth at $0 \mathrm{~K}$. The parameter $\alpha\left(X^{0}\right)$ obtained by a linear fit for experiments on tens of dots is in the range of $2 \mu \mathrm{eV} / \mathrm{K}$. This is larger than the value $\alpha\left(X^{0}\right) \simeq 0.5 \mu \mathrm{eV} / \mathrm{K}$ obtained by other groups on similar structures, ${ }^{11,12}$ but comparable to values measured in Ref. 19 We have measured the linewidth on a sample containing InAs dots grown under the same conditions but without a doped layer in the structure to check if the proximity of the back contact has any influence on this broadening, but the same value for $\alpha\left(X^{0}\right)$ was obtained.

Applying a more positive voltage to the gate, the dot can be charged with one additional electron. The twofold degenerate $s$ state is now fully occupied, to form $X^{1-}$. The spectra at 4.2 and $25 \mathrm{~K}$ resemble the $X^{0}$ transition very closely, see Fig. 2. The main change on increasing the temperature is again a strong deviation from a Lorentzian line shape. As for $X^{0}$, the $X^{1-}$ line is resolution limited at low temperature 
but increases with temperature. We estimate $\alpha\left(X^{1-}\right)$ $\simeq 3 \mu \mathrm{eV} / \mathrm{K}$. Our results show that the extra electron has very little effect on the line shape, although we consistently find $\alpha\left(X^{1-}\right)>\alpha\left(X^{0}\right)$ which reflects the larger number of carriers in $X^{1-}$ compared to $X^{0}$.

At more positive voltages again, the dot is charged with a total of three electrons creating $X^{2-}$, see Fig. 1. For $X^{2-}$ there are two final states after recombination: a triplet with a total electron spin of $S=1$ at higher energy (FWHM $<0.05 \mathrm{meV}$ at $4.2 \mathrm{~K}$ ) and a singlet $S=0$ at lower energy (FWHM $\sim 0.5 \mathrm{meV}$ at $4.2 \mathrm{~K}$ ), as can be seen in Fig. 1. The two transitions are split by the electron-electron exchange energy $2 X_{s p}^{e e},{ }^{3}$ where $X_{s p}^{e e}$ is the exchange interaction energy between an electron in the $s$ state and an electron in the $p$ state. At low temperature, recombination into the singlet state has a noticeably larger linewidth than recombination into the triplet state. This can be interpreted as evidence for a quick relaxation of the electron from the $p$ shell to the $s$ shell in the case of the singlet, introducing an uncertainty in energy for the final state of the PL transition, broadening the emission. In the triplet case, relaxation involves a spin flip, and the narrow linewidth for emission into the triplet state is evidence that the spin flip inhibits fast relaxation. The spin dependence of the relaxation clearly points to a phononmediated relaxation process, as the other possibility, an Auger interaction of quantum dot electrons with wetting layer electrons, would allow relaxation both with and without spin conservation. The electron $s$ to $p$ separation is about $30 \mathrm{meV}$ (Ref. 20) and therefore relaxation proceeds at $4 \mathrm{~K}$ by the emission of an optical phonon. The singlet-triplet splitting is typically $\sim 4 \mathrm{meV}$ (Refs. 3,21,22) (sample A) and $\sim 7 \mathrm{meV}$ (sample B). Recent experiments also show an additional fine structure: the triplet is not a single line, but a doublet separated by the electron-hole exchange energy of $\sim 0.2 \mathrm{meV}$ in sample B and $\sim 0.1 \mathrm{meV}$ in sample A. ${ }^{23}$ Although the electron-hole exchange is one order of magnitude smaller than the electron-electron exchange, it has to be taken into account when deconvoluting the measured spectra to analyze the FWHM of the $X^{2-}$ triplet transition as a function of temperature.

The linewidth for both triplet and singlet $X^{2-}$ emission increases surprisingly rapidly with temperature, see Fig. 3, at a rate much larger than for $X^{1-}$ and $X^{0}$. A comparison of Fig. 3 with Fig. 2 shows that at $30 \mathrm{~K}$, the $X^{2-}$ PL is considerably broader than the $X^{0}$ and $X^{1-}$ PL. We find that the values for $\alpha$ obtained from a linear fit to the data are now one order of magnitude larger for $X^{2-}$ than for $X^{1-}$, see Table I. $\alpha$ for the $X^{2-}$ singlet emission is consistently larger than for the $X^{2-}$ triplet emission (Table I).

The $X^{2-}$ has an occupied $p$ state whereas the $p$ state is unoccupied for $X^{1-}$ and $X^{0}$. This leads to the exchange-split $X^{2-}$ PL. We now consider the possible consequences of an occupied $p$ state on the broadening mechanisms in order to interpret the strong temperature dependence of the $X^{2-}$ PL.

Clearly, $X^{2-}$ has more carriers than $X^{0}$ and this will lead to a larger value of $\alpha$. However, the change on going from $X^{0}$ to $X^{1-}$ is modest and lies at the limit of our experimental resolution. A similar change can be expected on going from

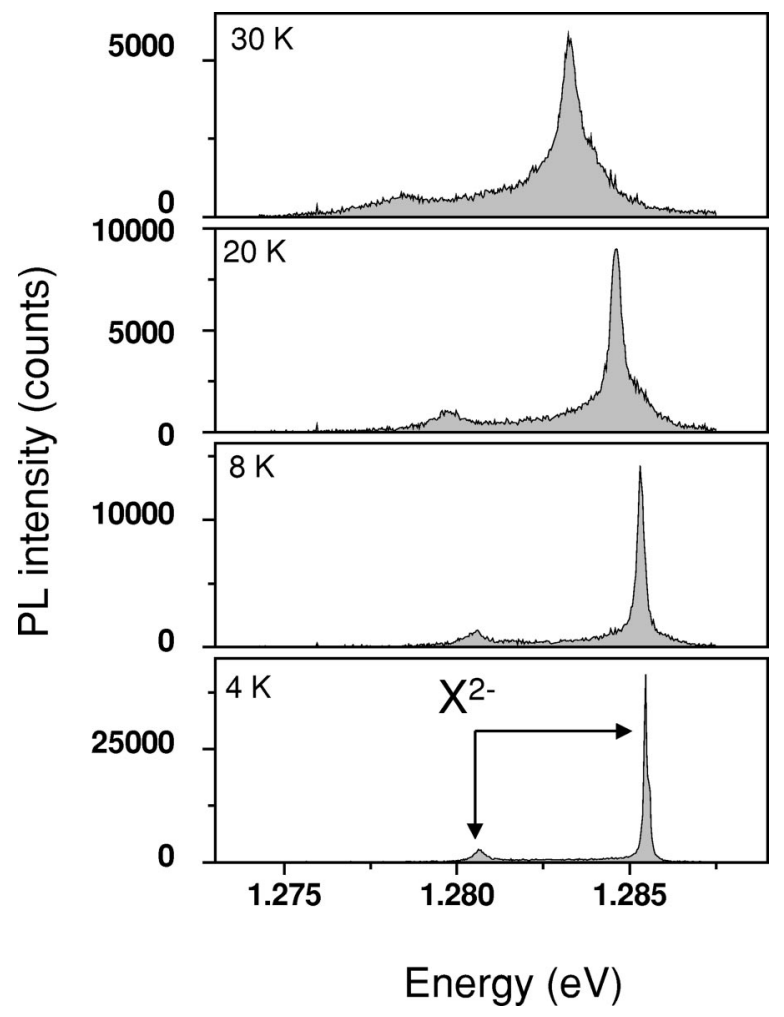

FIG. 3. The PL emission of the $X^{2-}$ transition at four different temperatures. Arrows indicate the emission into the $S=1 \quad(S=0)$ final state at high (low) energy.

$X^{1-}$ to $X^{2-}$ and this is clearly far smaller than the change we find experimentally. Therefore, a simple counting of particles cannot account for the large value of $\alpha$ for $X^{2-}$. The $p$ state couples to phonons differently to the $s$ state, and it might be thought that this can account for the enhanced temperature dependence. To verify this, we have applied the model of Besombes et al. ${ }^{13}$ an extension of the Huang Rhys theory of localized electron-phonon interaction ${ }^{24}$ to the exciton system in a quantum dot, to the different electron states. A similar model has been applied successfully by Cassabois et al. ${ }^{19}$ to explain the strong variation of $\Gamma_{0}$ and $\alpha\left(X^{0}\right)$ these authors have measured for different InAs quantum dots. In our case we have measured very different linewidths for the $X^{0}$ and $X^{2-}$ transitions for the same dot. We find that the matrix element for acoustic phonon scattering has a very similar magnitude for $p$-like states as for $s$-like states of the same

TABLE I. Examples of measured broadening parameters $\alpha$ for different states for dots from sample A (I-III) and B (IV), all values in $\mu \mathrm{eV} / \mathrm{K}$.

\begin{tabular}{lcccc}
\hline \hline Dot No. & $X^{0}$ & $X^{1-}$ & $X^{2-}(S=1)$ & $X^{2-}(S=0)$ \\
\hline I & 2.0 & 2.4 & 14.6 & 16.1 \\
II & 2.8 & 3.0 & 14.4 & 48.8 \\
III & & 4.4 & 15.8 & 42.4 \\
IV & & 1.2 & 20.0 & 36.0 \\
\hline abs. error & \pm 0.7 & \pm 0.7 & \pm 1.0 & \pm 3.5 \\
\hline \hline
\end{tabular}


quantum dot. The model of Besombes et al. can therefore not account for the strong change in linewidth we observe at elevated temperatures.

We have also considered the possibility that the $X^{2-}$ exciton is considerably more extended than the $X^{0}$ exciton, which would result in a different value of $\alpha$. However, this idea is not supported by the splitting in the $X^{2-}$ PL which measures the exchange energy $X_{s p}^{e e}=2.5 \mathrm{meV}$ for the dot in Fig. 1. This value is consistent with perturbation theory estimates which assume harmonic oscillator wave functions. ${ }^{17}$ The exchange energy therefore points to a well localized $p$ state. Also, measurements in magnetic field show that for dots which can be charged up to the $X^{2-}$ level, the $X^{2-}$ diamagnetic shift is the same as the $X^{0}$ diamagnetic shift (to within the experimental error of $10 \%){ }^{20}$ This too shows that the spatial extent of the $X^{2-}$ and $X^{0}$ excitons are almost the same. Therefore, the model of discrete dot levels interacting with acoustic phonons does not form the basis of an explanation for the observed $X^{2-}$ linewidths.

The $X^{2-}$ linewidths are influenced by relaxation of the final state, as explained above in the context of the PL spectrum at $4.2 \mathrm{~K}$. It could be argued that the relaxation rate increases strongly with increasing temperature causing the $X^{2-}$ PL to broaden. However, at $30 \mathrm{~K}$, the population of optical phonons is, as at $4 \mathrm{~K}$, insignificant, so optical phonon-mediated relaxation cannot have a strong temperature dependence in our temperature range. In any case, emission into the triplet also becomes broad at $30 \mathrm{~K}$, and if relaxation is responsible, a mechanism would have to arise which can flip the spin. Interpreting the broadening in terms of relaxation, the linewidth measured for triplet emission at $30 \mathrm{~K}$ would correspond to spin flip times of a few ps. This is orders of magnitude smaller than that previously measured in this temperature range ${ }^{5}$ and discussed in the literature. ${ }^{25,26}$ We consider therefore that a strong temperature dependence of the spin flip time to be an extremely unlikely explanation of the $X^{2-}$ temperature dependence. We are therefore forced to rule out a strong temperature dependence of the relaxation as an explanation for the temperature-induced broadening of the $X^{2-}$ PL.

A mechanism has been suggested recently ${ }^{16}$ to account for the broadening of excited quantum dot transitions observed in PLE experiments. ${ }^{14,15}$ The idea is that the excited quantum dot transitions are almost degenerate with so-called crossed transitions which involve for instance the quantum dot hole ground state and an electronic wetting layer state. An electron-hole pair in the excited quantum dot state can be scattered into the crossed state with an acoustic phonon, leading to a broadening of the transition. We note, however, that this mechanism cannot account for our results on the $X^{2-}$ because the electron configuration is in its ground state and the hole is also in its ground state. In fact, recombination occurs between an $s$ state hole and an $s$ state electron. Although the mechanism of Ref. 16 is not directly relevant here, we do believe, however, that continuum-like states are important for an explanation of our results.

For both samples A and B, $X^{3-}$ is the most highly charged exciton we can generate before charge spills out into the wetting layer, see Fig. 1. This suggests that the $p$ state is the

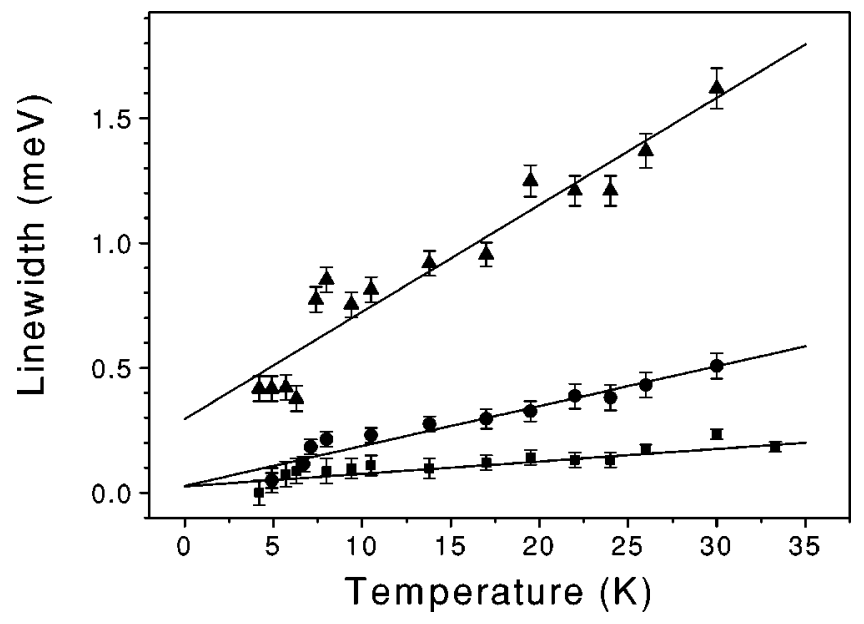

FIG. 4. Linewidth of the single dot PL transitions as a function of temperature for $X^{1-}$ (solid squares), the $X^{2-}$ triplet (circles), and singlet (solid triangles). The linewidths have been corrected for the resolution of the spectrometer-detector system. Linear fits to the data are shown as solid lines.

highest quantum dot state; at higher energies, there is no $d$ state but rather continuum states. The continuum states are associated with the wetting layer. The $p$ state lies considerably closer to the continuum states than the $s$ state, and this will cause $X^{2-}$ to be more strongly influenced by the continuum states than $X^{1-}$ and $X^{0}$. If there is an energy gap of, say, $10 \mathrm{meV}$ between the $p$ state and the continuum states, the additional contribution to the $X^{2-}$ broadening through a phonon-mediated interaction with the continuum would have an exponential dependence on temperature in the measured range $4-30 \mathrm{~K}$. This is not the case in the data of Fig. 4, where we observe a linear temperature dependence. In other words, our results do not support the idea of an energy gap between the quantum dot $p$ state and the continuum. Rather, the linear temperature dependence, typical of low-energy acoustic phonon scattering, suggests that there are states close in energy to the discrete quantum dot levels. From the form of the matrix element for acoustic phonon scattering, ${ }^{13}$ these states must be about $1 \mathrm{meV}$ or less away from the discrete quantum dot states to give the experimental linear increase in temperature. The picture that emerges is that the quantum dot density of states consists of a sharp $s$ state and a sharp $p$ state but with a broad background, close to zero at the energy of the $s$ state, but of significant magnitude at the $p$ state, as drawn schematically in Fig. 5. We suggest that the broad background in the density of states arises from the low-energy tail of the continuum. This picture also offers an explanation of the origin of the PL emission between the singlet and triplet transitions, as observed in Fig. 3. A $p$ electron in the initial state of $X^{2-}$ that is thermally excited, occupying a continuum state, will give rise to small electronelectron exchange energy. This results in an emission energy that lies between the singlet and triplet PL peaks.

A significant point is that for $X^{2-}$ the broadening of the emission into the singlet state is larger than the broadening of the emission into the triplet state. While this is related to spin at $4 \mathrm{~K}$, at $30 \mathrm{~K}$ it is due to a larger acoustic phonon scattering 


\section{Final State DOS}

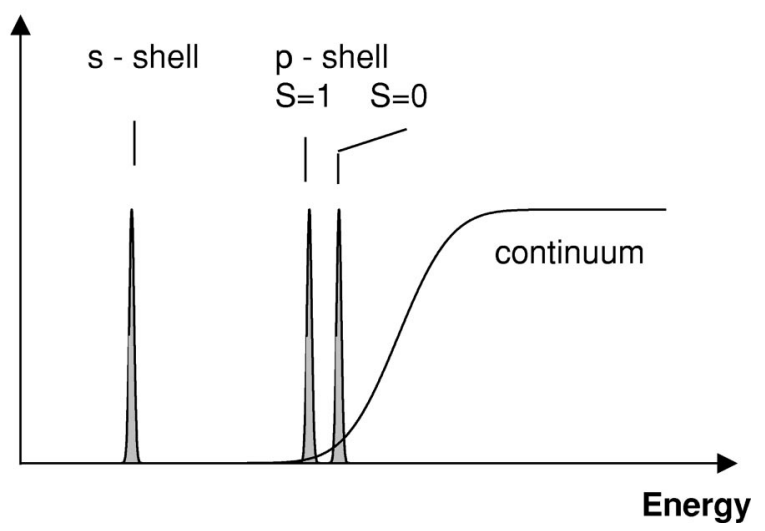

FIG. 5. Schematic drawing of the final state density of states (DOS) after photon emission with well defined $s$ and $p$ states. The $p$ states with total electron spin $S=1$ and $S=0$ are split by the electron-electron exchange. The low-energy tail of the continuum overlaps with the $p$ states, giving rise to the observed broadening of the $X^{2-}$ transition with temperature.

of the singlet state. The two $X^{2-}$ PL lines have the same initial state but different final states. This implies that the larger linewidth of the singlet emission relative to the triplet emission arises from a larger scattering rate of the singlet state. This is entirely consistent with the notion expressed above that the scattering arises with the tail of continuum states overlapping in energy with the localized quantum dot states. The singlet is at higher energy than the triplet (so that emission into the singlet is at lower energy than emission into the triplet) and therefore it seems reasonable to expect that the singlet state rides on a larger background density of states than the triplet. Within this model, most of the acoustic phonon scattering takes place in the final state because in the initial state, the hole pulls the energy levels down in energy by several $\mathrm{meV}$ and so further away from the continuum states.

To conclude, we have measured the temperature dependent linewidth for $X^{0}, X^{1-}$, and $X^{2-}$. We have found a drastically enhanced temperature dependence of the broadening of the $X^{2-}$ emission which we ascribe to the proximity of the $p$-like excited state to quantum dot continuum-like states. The continuum-like states can be occupied at elevated temperatures via scattering with acoustic phonons. We have focussed here on the specific case of the $X^{2-}$, for which our experiments show an overlap of continuum-like and discretelike features in the density of states. As the number and separation of discrete carrier levels is strongly dependent on the specific dot geometry, variations for different samples and materials can be expected. From the evidence presented here it can be predicted that the $X^{2-}$ PL should remain sharp in a very deep confining dot potential. We have demonstrated that embedding dots in a charge tunable structure is a powerful means of studying these details for a specific case, here InAs dots in GaAs. Our experiments show that only for deeply confined carriers the artificial atom model applies. The proximity of continuum and bound states will limit the transition linewidth as well as the effective carrier capture at elevated temperatures. This has to be taken into account when exploiting the discrete nature of quantum dot transitions for future device applications.

This work was financially supported by EPSRC (UK) and DFG (Grant No. SFB348).
${ }^{1}$ D. Gammon and D.G. Steel, Phys. Today 55, 36 (2002).

${ }^{2}$ M. Bayer, O. Stern, P. Hawrylak, S. Fafard, and A. Forchel, Nature (London) 405, 923 (2000).

${ }^{3}$ R.J. Warburton, C. Schäflein, D. Haft, F. Bickel, A. Lorke, K. Karrai, J. Garcia, W. Schoenfeld, and P.M. Petroff, Nature (London) 405, 926 (2000).

${ }^{4}$ P. Borri, W. Langbein, S. Schneider, U. Woggon, R.L. Sellin, D. Ouyang, and D. Bimberg, Phys. Rev. Lett. 87, 157401 (2001).

${ }^{5}$ M. Paillard, X. Marie, P. Renucci, T. Amand, A. Jbeli, and J. Gérard, Phys. Rev. Lett. 86, 1634 (2001).

${ }^{6}$ P. Michler, A. Kiraz, C. Becher, W.V. Schoenfeld, P.M. Petroff, L. Zhang, E. Hu, and A. Imamoglu, Science 290, 2282 (2000).

${ }^{7}$ E. Moreau, I. Robert, L. Manin, V. Thierry-Mieg, J.M. Gérard, and I. Abram, Phys. Rev. Lett. 87, 183601 (2001).

${ }^{8}$ Z. Yuan, B.E. Kardynal, R.M. Stevenson, A.J. Shields, C.J. Lobo, K. Cooper, N.S. Beattie, D.A. Ritchie, and M. Pepper, Science 295, 102 (2001).

${ }^{9}$ C. Santori, D. Fattal, J. Vucković, G. Solomon, and Y. Yamamoto, Nature (London) 419, 594 (2002).

${ }^{10}$ K. Matsuda, K. Ikeda, T. Saiki, H. Tsuchiya, H. Saito, and K. Nishi, Phys. Rev. B 63, 121304 (2001).

${ }^{11}$ M. Bayer and A. Forchel, Phys. Rev. B 65, 041308 (2002).
${ }^{12}$ C. Kammerer, G. Cassabois, C. Voisin, M. Perrin, C. Delalande, P. Roussignol, and J.M. Gérard, Appl. Phys. Lett. 81, 2737 (2002).

${ }^{13}$ L. Besombes, K. Kheng, L. Marsal, and H. Mariette, Phys. Rev. B 63, 155307 (2001).

${ }^{14}$ Y. Toda, O. Moriwaki, M. Nishioka, and Y. Arakawa, Phys. Rev. Lett. 82, 4114 (1999).

${ }^{15}$ C. Kammerer, G. Cassabois, C. Voisin, C. Delalande, P. Roussignol, A. Lemaitre, and J.M. Gérard, Phys. Rev. B 65, 033313 (2001).

${ }^{16}$ A. Vasanelli, R. Ferreira, and G. Bastard, Phys. Rev. Lett. 89, 216804 (2002).

${ }^{17}$ R.J. Warburton, B.T. Miller, C.S. Dür, C. Bödefeld, K. Karrai, J.P. Kotthaus, G. Medeiros-Ribeiro, P.M. Petroff, and S. Huant, Phys. Rev. B 58, 16221 (1998).

${ }^{18}$ A. Högele, S. Seidl, R. J. Warburton, K. Karrai, and P. M. Petroff (unpublished).

${ }^{19} \mathrm{G}$. Cassabois (private communication).

${ }^{20}$ C. Schulhauser, D. Haft, R.J. Warburton, K. Karrai, A.O. Govorov, A.V. Kalameitsev, A. Chaplik, W. Schoenfeld, J. Garcia, and P.M. Petroff, Phys. Rev. B 66, 193303 (2002).

${ }^{21}$ J.J. Finley, P.W. Fry, A.D. Ashmore, A. Lemaitre, A.I. Tartak- 
ovskii, R. Oulton, D.J. Mowbray, M.S. Skolnick, M. Hopkinson, P.D. Buckle, and P.A. Maksym, Phys. Rev. B 63, 161305 (2001).

${ }^{22}$ F. Findeis, M. Baier, A. Zrenner, M. Bichler, G. Abstreiter, U. Hohenester, and E. Molinari, Phys. Rev. B 63, 121309 (2001).

${ }^{23}$ B. Urbaszek, R.J. Warburton, K. Karrai, B.D. Gerardot, P.M. Petroff, and J.M. Garcia, Phys. Rev. Lett. 90, 247403 (2003).
${ }^{24}$ C. Duke and G.D. Mahan, Phys. Rev. 139, A1965 (1965).

${ }^{25}$ I.A. Merkulov, A.L. Efros, and M. Rosen, Phys. Rev. B 65, 205309 (2002).

${ }^{26}$ E. Tsitsishvili, R.v. Baltz, and H. Kalt, Phys. Rev. B 66, 161405 (2002). 